\title{
Uniqueness of solutions of linear relay systems ${ }^{1}$
}

\author{
Y. J. Lootsma ${ }^{\text {a, }}$, A. J. van der Schaft ${ }^{\mathrm{a}, \mathrm{b}}$, M. K. Çamlıbel ${ }^{\mathrm{c}, 2}$ \\ ${ }^{a}$ Faculty of Mathematical Sciences, University of Twente, P.O. Box 217, 7500 AE Enschede, Netherlands \\ ${ }^{\mathrm{b}}$ CWI, P.O. Box 94079, 1090 GB Amsterdam, The Netherlands \\ 'Department of Control and Computer Eng., Istanbul Technical University, 80626 Istanbul, Turkey
}

Received 13 October 1997; revised 4 June 1998; received in final form 22 September 1998

Using the constructive theory of the Linear Complementarity Problem, conditions are given for uniqueness of solutions of the hybrid dynamics in linear relay systems.

\begin{abstract}
Conditions are given for uniqueness of solutions of linear time-invariant systems under relay feedback. From a hybrid dynamical point of view this entails the deterministic specification of the discrete transition rules. The results are based on the formulation of relay systems as complementarity systems, and use the constructive theory of the Linear Complementarity Problem. (C) 1999 Elsevier Science Ltd. All rights reserved.
\end{abstract}

Keyword: Hybrid systems; Relays; Well-posedness; Complementarity problems; Coulomb friction

\section{Introduction}

In this paper we consider a special type of hybrid dynamics as occurring in linear dynamical systems containing ideal relay elements. The behavior of an ideal relay (see Fig. 1) is given by three modes of operation

(i) $f \geq 0, e=c_{1}$,

(ii) $f \leq 0, e=-c_{2}$,

(iii) $f=0,-c_{2} \leq e \leq c_{1}$.

Such relay characteristics appear in various areas of engineering. They serve as an idealized model of Coulomb friction (with $f$ being the velocity and $e$ being the Coulomb force). Within a control context various (physical) relay elements have been discussed in the literature,

*Corresponding author. Tel.: + 31534893453 ; fax: + 31534340733 ; e-mail: Y. J. Lootsma@math.utwente.nl.

${ }^{1}$ This paper was not presented at any IFAC meeting. This paper was recommended for publication in revised form by Guest Editors J. M. Schumacher, A. S. Morse, C. C. Pantelides, and S. Sastry.

${ }^{2}$ Currently visiting CWI, P.O. Box 94079,1090 GB Amsterdam, Netherlands. see especially (Tsypkin, 1984). Furthermore, switching control schemes such as

$u=\left\{\begin{aligned}-1 & \text { if } y>0 \\ 1 & \text { if } y<0\end{aligned}\right.$

(with $y$ the output and $u$ the input of a control system) lead to a relay characteristic (with $f=y$ and $e=-u$ ), by using Filippov's solution concept (Filippov, 1988) of equivalent control or convex definition for $y=0$. (We will briefly return to Filippov's solution concept later on.)

From the point of view of dynamics, a fundamental problem of systems containing ideal relay elements is that existence and uniqueness of solutions is not guaranteed. An example of a system exhibiting non-uniqueness of solutions is the following:

$$
\begin{aligned}
& \dot{x}_{1}(t)=x_{2}(t), \quad y(t)=x_{2}(t) \\
& \dot{x}_{2}(t)=-x_{1}(t)-u(t)
\end{aligned}
$$

with,

$$
\begin{array}{ll}
u(t)=-1 & \text { if } y(t)>0 \\
u(t)=1 & \text { if } y(t)<0, \\
-1 \leq u(t) \leq 1 & \text { if } y(t)=0 .
\end{array}
$$




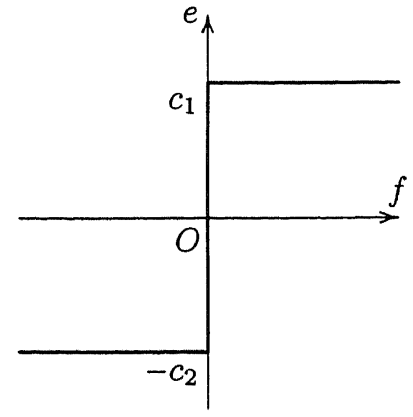

Fig. 1. Characteristic of an ideal relay.

(This could be interpreted as a mass--spring system subject to a "reversed" - and thus non-physical Coulomb friction.) In this example, from any initial state $x(0)=\left(x_{1}(0), x_{2}(0)=(c, 0)\right.$, with $|c|<1$, there are three possible (smooth) initial solutions for $t \in[0, \varepsilon[, \varepsilon>0$, that are allowed by the equations and inequalities above:

(i) $u(t)=1, \quad x_{1}(t)=\left(x_{1}(0)+1\right) \cos (t)-1$,

$x_{2}(t)=y(t)=-\left(1+x_{1}(0)\right) \sin (t)<0$,

(ii) $u(t)=-1, \quad x_{1}(t)=\left(x_{1}(0)-1\right) \cos (t)+1$,

$x_{2}(t)=y(t)=\left(1-x_{1}(0)\right) \sin (t)>0$,

(iii) $u(t)=-x_{1}(0) \in[-1,1], \quad x_{1}(t)=x_{1}(0)$,

$x_{2}(t)=y(t)=0$.

So the above system (1) is not well-posed as a dynamical system. If the sign in front of $u$ in the first equation of $(1)$ is reversed however (and thus physically the Coulomb friction has the correct sign!), there is only one smooth solution from every initial state $x_{0}$, as will follow from the main theorem of the present paper.

By associating three discrete states ("locations" or "modes") to the three linear parts of the relay characteristic, one can view (1) as a hybrid dynamical system. The three possible smooth solutions (i) (iii) in the above example exactly correspond to these three discrete states. Seen from this point of view the hybrid dynamical system (1) serves as a clear example where the discrete state may not be uniquely determined by the continuous state. (See for a different type of example Barton and Pantelides, 1994.) While for general hybrid dynamical systems such a subordination of the discrete state to the continuous state is not necessary at all (even to the contrary!), for relay systems such as (1) this is a very desirable property. Indeed, since only the three locations (discrete states) together with their invariants are given, while the specification of the transition rules from one location to another is completely left open, the system equations are only well posed if they admit only one "acceptable" full specification of the hybrid dynamics. (In general, it seems not reasonable to assign a non-deterministic behavior to relay systems - think for example of a mechanical system with Coulomb friction. Nevertheless, the classical Painlevé example, as described e.g. in Brogliato (1996) and Lötstedt (1981), does exhibit non-uniqueness of solutions.)

Of course, the above example containing a single relay element is easy to interpret by noting that for uniqueness of solutions one needs the "correct", that is, negative, feedback sign. A discussion of this phenomenon can be found e.g. in Filippov (1988) and Utkin (1992). Nevertheless, for systems containing multiple relay-elements, providing conditions for uniqueness of solutions is not at all trivial, and the present paper seems to be the first in doing this. Furthermore, even if one knows (or trusts) the system has unique solutions then the actual computation of this solution may be far from easy, especially in the multiple relay case. The main problem is precisely in computing the "discrete part" of the hybrid dynamics (the transitions from one location to another), since they are not a priori specified by the system equations of the relay system. Certainly for simulation purposes this is an important topic (see Mattsson, 1996; Cellier et al., 1993 for a discussion of the problems which already arise in single relay systems). In the context of simulation of mechanical systems with multiple Coulomb friction elements, this computational issue has been studied intensively, see e.g. Lötstedt (1981) and Glocker and Pfeiffer (1993)

We emphasize at this point that we only consider ideal relay elements. That means that we do not treat hysteresis effects as usually occur in physical relay elements, although it seems worthwhile to interpret our results for the limiting behavior when the hysteresis gap tends to zero (see e.g. Seidman, 1995, for studies in this area). Furthermore, we do not treat Coulomb friction with higher break-off friction than the slip friction, as is sometimes considered in the modeling of dry friction (see e.g. Cellier et al., 1993; Mattsson, 1996).

In the present paper we will derive sufficient conditions for uniqueness of solutions of linear time-invariant dynamical systems containing multiple (ideal) relay elements. The main tool is the theory of the Linear Complementarity Problem (LCP) from optimization theory, see Cottle et al. (1992). The work can be regarded as a continuation of the work on complementarity hybrid systems (Van der Schaft and Schumacher, 1996, 1998; Heemels et al., 1997), where the LCP was used for analyzing the dynamics of (possibly nonlinear) systems containing "ideal diode characteristics" $e \geq 0, f \geq 0, e f=0$. In fact, in Van der Schaft and Schumacher (1998) it was already shown how systems with relay elements can be represented as complementarity systems. The theory of existence and uniqueness for complementarity systems as developed in Van der Schaft and Schumacher (1996, 1998) and Heemels et al. (1997) does not apply, however, to the class of complementarity systems arising from relay systems. We will show that for these relay systems, contrary 
to the "ideal diode" case considered in Van der Schaft and Schumacher (1996, 1998) and Heemels et al. (1997), the continuous-state part of the unique solution is continuous as a function of time. This means that the switching from one location (mode) to the other does not entail a reinitialization of the continuous-state part of the system. Because of this the technical difficulties of the generalized (distributional) solution concept for complementarity systems as described in Heemels et al. (1997) and Van der Schaft and Schumacher (1996) can be completely avoided.

A major advantage in the use of the LCP is that, apart from giving elegant sufficient conditions for uniqueness of solutions, it also provides a strong framework for actually computing the unique solution. In fact, we believe that efficient simulation routines can be based on our approach. The usefulness of the LCP in computing solutions of mechanical systems with Coulomb friction has been realized before, at least starting with the work of Lötstedt (1981) and continued by various authors, see e.g. Glocker and Pfeiffer (1993) and the references quoted in Brogliato (1996). The LCP-formulation of systems with Coulomb friction employed in these papers is however different from the formulation in present paper, and does not seem to lead to simple uniqueness criteria. (Note also that in these papers the maximal friction force is not taken to be constant, but is a function of the normal constraint force. The systems under consideration are therefore more complex than in the present paper; moreover they are nonlinear.)

\section{Linear relay systems as complementarity systems}

Consider an arbitrary (explicit) linear dynamics containing $m$ relay elements (and no external inputs). By first extracting the $m$ relay elements, and assigning to every "port" created in this manner an input and an output variable $\bar{u}_{i}$, resp. $\bar{y}_{i}$, it is readily seen that such a system can be represented as in Fig. 2.

Here, the input-state-output system $P$ is given by

$\dot{x}(t)=A x(t)+B \bar{u}(t), \quad x(t) \in \mathbb{R}^{n}, \bar{u}(t) \in \mathbb{R}^{m}$,

$\bar{y}(t)=C x(t)+D \bar{u}(t), \quad \bar{y}(t) \in \mathbb{R}^{m}$

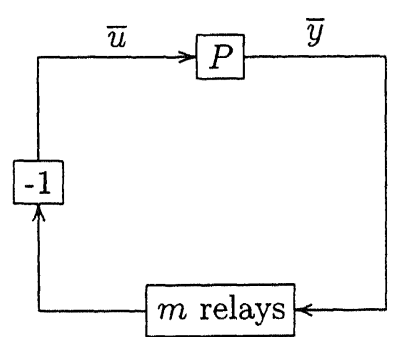

Fig. 2. Feedback system with $m$ ideal relays.

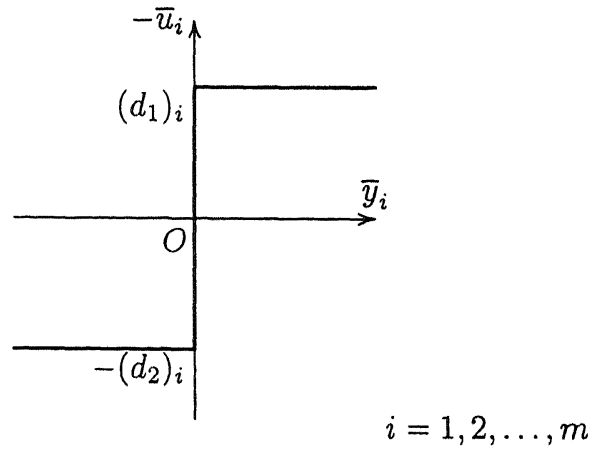

Fig. 3. The characteristics of the $m$ ideal relays.

The matrices $A, B, C$ and $D$ are given matrices of sizes $n \times n, n \times m, m \times n$ and $m \times m$, respectively.

The block " $m$ relays" denotes $m$ ideal relays with characteristics as given in Fig. 3. The numbers $\left(d_{1}\right)_{i}$ and $\left(d_{2}\right)_{i}$, $i=1, \ldots, m$, in this figure are the components of the constant vectors $d_{1}$ and $d_{2} \in \mathbb{R}^{m}$ satisfying

$d_{1} \geq 0, \quad d_{2} \geq 0, \quad d_{1}+d_{2}>0$.

Define as in Van der Schaft and Schumacher (1998)

$u(t)=\left(\begin{array}{l}u_{a}(t) \\ u_{b}(t)\end{array}\right)$,

as

$u_{a}(t)=d_{1}+\bar{u}(t)$,

$u_{h}(t)=d_{2}-\bar{u}(t)$,

and define $y(t)$ by

$y(t)=\left(\begin{array}{l}y_{a}(t) \\ y_{b}(t)\end{array}\right)=\left(\begin{array}{l}(\bar{y}(t))^{+} \\ (y(t))^{-}\end{array}\right)$,

where $(\bar{y}(t))^{+}$is the non-negative part of vector $\bar{y}(t)$, and $(l(t))^{-}$is the non-positive part of this vector.

The relay system in Fig. 2 can then be described by Eqs. (2)-(5), together with the complementarity constraints

$y(t)=\left(\begin{array}{l}y_{a}(t) \\ y_{b}(t)\end{array}\right) \geq 0$,

$u(t)=\left(\begin{array}{l}u_{a}(t) \\ u_{b}(t)\end{array}\right) \geq 0$

$u^{\mathrm{T}}(t) y(t)=0$.

Such systems have been called "complementarity systems" in Van der Schaft and Schumacher $(1996,1998)$.

From Eq. (6) we see that for every $i=1,2, \ldots, 2 m$ either $(u(t))_{i}$ or $(y(t))_{i}$ is zero (or both). It is however not possible that $\left(u_{a}(t)\right)_{i}=\left(u_{b}(t)\right)_{i}=0$, for it follows from Eqs. (4) and (3) that

$\left(u_{a}(t)\right)_{i}+\left(u_{b}(t)\right)_{i}=\left(d_{1}+d_{2}\right)_{i}>0$. 
From this we conclude that for every $i=1,2, \ldots, m$, we have

$\left(u_{a}(t)\right)_{i}>0$ or $\left(u_{b}(t)\right)_{i}>0$.

Note that this implies, in accordance with Eq. (5), that $\left(y_{a}(t)\right)_{i}=0$ or $\left(y_{b}(t)\right)_{i}=0$.

The set of Eqs. (2)-(6) thus defines a hybrid system with in principle $2^{2 m}$ different locations (modes) corresponding to the equalities

$u_{i}=0 \quad$ or $\quad y_{i}=0, \quad i=1,2, \ldots, 2 m$.

However, because of Eq. (7), we see that the modes with $\left(u_{a}\right)_{i}=0$ and $\left(u_{b}\right)_{i}=0$ for some $i$ are void, thereby leaving us with $3^{m}$ modes, in accordance with the three-mode characteristic of the ideal relay element.

Remark 1. For some applications it is useful to generalize the relay characteristics of Fig. 3 to the more general characteristics as depicted in Fig. 4.

Here $\alpha, d_{1}, d_{2} \in \mathbb{R}^{m}$ only need to satisfy the requirement $\left(d_{1}+d_{2}\right)>0$.

This still can be modeled within the complementarity framework as follows. If we apply the coordinate transformation

$\tilde{\imath}=\bar{u}+\frac{1}{2}\left(d_{1}-d_{2}\right)$,

ve find

$\dot{x}(t)=A x(t)+B \tilde{u}(t)-\frac{1}{2} B\left(d_{1}-d_{2}\right)$,

$\bar{y}(t)=C x(t)+D \tilde{u}(t)-\frac{1}{2} D\left(d_{1}-d_{2}\right)$.

Now, defining

$y(t)=\left(\begin{array}{l}y_{a}(t) \\ y_{b}(t)\end{array}\right)=\left(\begin{array}{l}(\bar{y}(t)-\alpha)^{+} \\ (\bar{y}(t)-\alpha)^{-}\end{array}\right)$,

and

$u(t)=\left(\begin{array}{l}u_{a}(t) \\ u_{b}(t)\end{array}\right)=\left(\begin{array}{l}\frac{1}{2}\left(d_{1}+d_{2}\right)+\tilde{u}(t) \\ \frac{1}{2}\left(d_{1}+d_{2}\right)-\tilde{u}(t)\end{array}\right)$,

the characteristics are described by Eqs. (6), (8)-(11). It is straightforward to extend the results derived in this paper to these generalized characteristics.

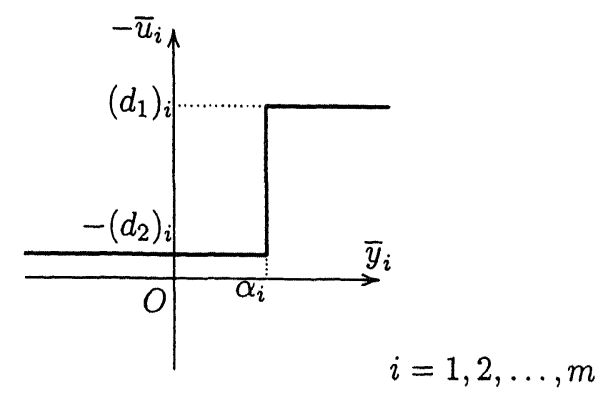

Fig. 4. More general relay characteristics.

\section{A frequency-domain approach}

Continuing upon the work in Van der Schaft and Schumacher (1998) and Heemels et al. (1997) we will study existence and uniqueness of the solutions of complementarity system (2)-(6) by transforming the equations to the frequency domain.

Let $W(s)$ be a strictly proper rational function in the complex variable $s$ with real coefficients. To this function we can associate the coefficients $w^{j}$ of its power series expansion around infinity

$W(s)=\frac{w^{0}}{s^{1}}+\frac{w^{1}}{s^{2}}+\frac{w^{2}}{s^{3}}+\cdots$

The corresponding real-analytic time function $w(t)$ obtained by inverse Laplace transformation is then given by

$w(t)=w^{0}+w^{1} t+\frac{1}{2 !} w^{2} t^{2}+\frac{1}{3 !} w^{3} t^{3}+\cdots$.

Both the conditions $W(s) \geq 0$ for sufficiently large $s \in \mathbb{R}$ and $w(t) \geq 0$ for $t \in[0, \varepsilon[, \varepsilon>0$ sufficiently small, are then equivalent to the condition that either all $w^{i}(i=0,1, \ldots)$ are zero or the first nonzero element of $\left(w^{0}, w^{1}, w^{2}, \ldots\right)$ is positive.

The relay system described in the previous section can be rewritten to the frequency domain as follows. By taking the Laplace transform of Eq. (2), we get

$\bar{Y}(s)=\left(C\left(s I_{n}-A\right)^{-1} B+D\right) \bar{U}(s)+C\left(s I_{n}-A\right)^{-1} x_{0}$,

where $x_{0}=x(0)$ is the initial state of the system. The Laplace transforms of Eqs. (4) and (5) are given by

$U_{a}(s)=\frac{1}{s} d_{1}+\bar{U}(s)$

$U_{b}(s)=\frac{1}{s} d_{2}-\bar{U}(s)$

respectively,

$Y(s)=\left(\begin{array}{l}Y_{a}(s) \\ Y_{b}(s)\end{array}\right)=\left(\begin{array}{l}(\bar{Y}(s))^{+} \\ (\bar{Y}(s))^{-}\end{array}\right)$.

Condition (6) is in the frequency domain replaced with

$\left.\begin{array}{l}Y(s) \geq 0 \\ U(s) \geq 0\end{array}\right\}$ for sufficiently large $s \in \mathbb{R}$

$U^{\mathrm{T}}(s) Y(s)=0$.

Furthermore, with the notation

$T(s) \stackrel{\text { Def }}{=} C\left(s I_{n}-A\right)^{-1}$,

$G(s) \stackrel{\text { Def }}{=} C\left(s I_{n}-A\right)^{-1} B+D$, 
The equalities (14)-(16) can be rewritten as

$$
\begin{aligned}
{\left[\begin{array}{l}
U_{a}(s) \\
U_{b}(s)
\end{array}\right]=} & {\left[\begin{array}{r}
-G^{-1}(s) T(s) x_{0}+\frac{1}{s} d_{1} \\
G^{-1}(s) T(s) x_{0}+\frac{1}{s} d_{2}
\end{array}\right] } \\
& +\left[\begin{array}{rr}
G^{-1}(s) & -G^{-1}(s) \\
-G^{-1}(s) & G^{-1}(s)
\end{array}\right]\left[\begin{array}{r}
Y_{a}(s) \\
Y_{b}(s)
\end{array}\right]
\end{aligned}
$$

or

$U(s)=q(s)+M(s) Y(s)$,

where $q(s)$ and $M(s)$ are given by

$$
\begin{aligned}
& q(s)=\left[\begin{array}{r}
-G^{-1}(s) T(s) x_{0}+\frac{1}{s} d_{1} \\
G^{-1}(s) T(s) x_{0}+\frac{1}{s} d_{2}
\end{array}\right], \\
& M(s)=\left[\begin{array}{rr}
G^{-1}(s) & -G^{-1}(s) \\
-G^{-1}(s) & G^{-1}(s)
\end{array}\right] .
\end{aligned}
$$

For constant $s \in \mathbb{R}$ sufficiently large, the set of Eqs. (18) together with complementarity conditions (17) is known as a Linear Complementarity Problem. For completeness we recall from Cottle et al. (1992) the formulation of the Linear Complementarity Problem (LCP).

Linear Complementarity Problem $(\operatorname{LCP}(q, M))$. Given a matrix $M \in \mathbb{R}^{n \times n}$ and a vector $q \in \mathbb{R}^{n}$, find $w, z \in \mathbb{R}^{n}$ such that

$w=q+M z$,

$w \geq 0, z \geq 0$,

$z^{\mathrm{T}} w=0$

or show that no such vectors $w, z$ exist.

In this definition (and in the rest of this paper), the inequalities should be considered to hold componentwise. The inequality $x \geq y, x, y \in \mathbb{R}^{n}$, means $x_{i} \geq y_{i}$, $i=1,2, \ldots, n$, while $x>y$ means $x_{i}>y_{i}, i=1,2, \ldots, n$. A vector $z$ satisfying the inequalities $z \geq 0$ and $q+M z \geq 0$ is said to be feasible. The $\operatorname{LCP}(q, M)$ is said to be feasible if a feasible vector exists. The $\operatorname{LCP}(q, M)$ is said to be solvable if it has a solution (Cottle et al., 1992).

We introduce some further definitions and recall a basic result concerning the LCP (see e.g. Cottle et al., 1992):

Definition 2. Let $M \in \mathbb{R}^{m \times n}$ be given. For index sets $I \subseteq\{1,2, \ldots, m\}$ and $J \subseteq\{1,2, \ldots, n\}$, the submatrix $M_{I J}$ of $M$ is the matrix whose entries lie in the rows of $M$ indexed by $I$ and the columns indexed by $J$. If $I=\left\{1,2, \ldots, m_{\}}\right.$, we denote $M_{I J}$ by $M_{J_{J}}$; similarly, if $J=\{1,2, \ldots, n\}$, we denote $M_{I J}$ by $M_{I}$.

Definition 3. Given a matrix $M \in \mathbb{R}^{n \times n}$ and two nonempty subsets $I$ and $J$ of $\{1,2, \ldots, n\}$ of equal cardinality, the $(I, J)$-minor of $M$ is the determinant of the square submatrix $M_{I J} \stackrel{\text { Def }}{=}\left(m_{i j}\right)_{i \in I, j \in J}$. The principal $m i$ nors are those with $I=J$.

Definition 4. A matrix $M \in \mathbb{R}^{n \times n}$ is said to be a $P$-matrix if all its principal minors are positive. $M$ is said to be a $\boldsymbol{P}_{0}$-matrix if all its principal minors are non-negative.

Theorem 5 (see e.g. Theorem 3.3 .7 in Cottle et al., 1992). A matrix $M \in \mathbb{R}^{n \times n}$ is a $\boldsymbol{P}$-matrix if and only if the $\operatorname{LCP}(q, M)$ has a unique solution for all vectors $q \in \mathbb{R}^{n}$.

Remark 6. An attempt to interpret the notion of a $\boldsymbol{P}$ matrix is the following. It can be readily seen that the equation $w=q+M z$ has for all index sets $I \subset\{1, \ldots, n\}$ a unique solution $w, z \in \mathbb{R}^{n}$ with $w_{i}=0, i \in I$, and $z_{j}=0$, $j \notin I$, if and only if the principal minors of $M$ are all non-zero. By enforcing this condition to positive principal minors one ensures the existence of a unique solution $w, z \in \mathbb{R}^{n}$ satisfying additionally $w \geq 0, z \geq 0$, with $I$ being determined by $q$. (One may also note, see e.g. Cottle et al. (1992, p. 147), that a symmetric matrix is a $\boldsymbol{P}$-matrix if it is positive definite and one only needs to check positivity of the leading principal minors.)

How do we solve the $\operatorname{LCP}(q(s), M(s))$, with $q(s)$ and $M(s)$ as in (20), for $s \in \mathbb{R}$ sufficiently large? First, we note that we cannot use Theorem 3 directly since $\operatorname{det} M(s)=0$. Or the other hand, the special structure of $M(s)$ allows us tc write

$M(s)=\left[\begin{array}{r}I_{m} \\ -I_{m}\end{array}\right] G^{-1}(s)\left[\begin{array}{ll}I_{m} & -I_{m}\end{array}\right]$.

and to relate the properties of $M(s)$ directly with those of $G(s)$, as will follow from the next technical lemmas.

Lemma 7. If $H \in \mathbb{R}^{k \times k}$ is a P-matrix, then $H^{-1}$ is a $\boldsymbol{P}$ matrix.

Proof. According to Theorem 3.3.4 in Cottle et al. (1992), the following two statements are equivalent:

(i) $M$ is a $P$-matrix,

(ii) $\left[z_{i}(M z)_{i} \leq 0\right.$ for all $\left.i\right] \Rightarrow[z=0]$.

Let $H$ be a $P$-matrix. Let $z \in \mathbb{P}^{k}$, define $y$ by $y=H^{-1} z$, then

$z_{i}\left(H^{-1} z\right)_{i}=(H y)_{i}\left(H^{-1} H y\right)_{i}=y_{i}(H y)_{i} \leq 0$ for all $i$.

Since $H$ is a $\boldsymbol{P}$-matrix, it follows that $y=0$, hence $z=0$, and thus $H^{-1}$ is a $P$-matrix.

Lemma 8. Let $H \in \mathbb{R}^{k \times k}$ be a P-matrix and $M=\left[\begin{array}{ll}I_{k} & -I_{k}^{T}\end{array}\right]^{\mathrm{T}} H\left[\begin{array}{ll}I_{k} & -I_{k}\end{array}\right]$.

Then we have the following statements:

(1) A principal minor of $M$ is either a principal minor of H or zero. 
(2) $M$ is a $\boldsymbol{P}_{0}$-matrix.

(3) For each index set $\alpha$ with det $M_{\alpha \alpha}=0$, the columns of $M_{\cdot \alpha}$ are linearly dependent.

Proof. Let $\alpha \subseteq\{1,2, \ldots, 2 k\}$ be an index set. By decomposing $\alpha$ into two index sets, we can write

$\alpha=I \cup J, \quad I \subseteq\{1,2, \ldots, k\}, J \subseteq\{k+1, k+2, \ldots, 2 k\}$.

Define $\hat{J}=\{i \mid i+k \in J\}, \quad \tilde{\alpha}=I \cup \hat{J}, \quad n_{1}=\operatorname{card} I \quad$ and $n_{\jmath}=$ card $\hat{J}$. If $\alpha=I$ or $\alpha=J$, it follows directly from the definition of $M$ that

$\operatorname{det} M_{\alpha \alpha}=\operatorname{det} H_{\tilde{\alpha} \tilde{\alpha}}$.

If $I \neq \emptyset$ and $J \neq \emptyset$, then it can be easily checked that

$$
\begin{aligned}
M_{\alpha \alpha} & =\left[\begin{array}{ll}
M_{I I} & M_{I J} \\
M_{J I} & M_{J J}
\end{array}\right] \\
& =\left[\begin{array}{rr}
H_{I I} & -H_{I \hat{J}} \\
-H_{\hat{J} I} & H_{\hat{J} \hat{J}}
\end{array}\right] \\
& =\left[\begin{array}{ll}
I_{n_{I}} & O_{n_{I} \times n_{\hat{J}}} \\
O_{n_{\hat{J}} \times n_{I}} & -I_{n_{\hat{J}}}
\end{array}\right]\left[\begin{array}{ll}
H_{I I} & H_{I \hat{J}} \\
H_{\hat{J} I} & H_{\hat{J} \hat{J}}
\end{array}\right]\left[\begin{array}{cc}
I_{n_{I}} & O_{n_{I} \times n_{\hat{J}}} \\
O_{n_{\hat{j}} \times n_{l}} & -I_{n_{\hat{J}}}
\end{array}\right]
\end{aligned}
$$

and, consequently,

$\operatorname{det} M_{\alpha \alpha}= \begin{cases}\operatorname{det} H_{\dot{\alpha} \grave{x}} & \text { if } \operatorname{I\cap } \hat{J}=\emptyset, \\ 0 & \text { if } I \cap \widehat{J} \neq \emptyset .\end{cases}$

It is now obvious that $M$ is a $\boldsymbol{P}_{0}$-matrix and if $\operatorname{det} M_{\alpha x}=0$, then the columns of $M_{\alpha \alpha}$ are linearly dependent.

Lemma 9. Let $H \in \mathbb{R}^{k \times k}$ be a P-matrix and

$M=\left[\begin{array}{ll}I_{k} & -I_{k}\end{array}\right]^{\mathrm{T}} H\left[\begin{array}{ll}I_{k} & -I_{k}\end{array}\right]$.

It follows that

$z_{i}\left(M^{\mathrm{T}} z\right)_{i} \leq 0$ for all $i=1,2, \ldots, 2 k \Rightarrow$

$z_{i}\left(M^{\mathrm{T}} z\right)_{i}=0$ for all $i=1,2, \ldots, 2 k$.

Proof. Assume that $z_{i}\left(M^{\mathrm{T}} z\right)_{i} \leq 0$ for all $i=1,2, \ldots, 2 k$. Let

$z=\left(\begin{array}{l}u \\ v\end{array}\right)$

with $u, v \in \mathbb{R}^{k}$.

$M^{\mathrm{T}} z=\left[\begin{array}{ll}I_{k} & -I_{k}\end{array}\right]^{\mathrm{T}} H^{\mathrm{T}}\left[\begin{array}{ll}I_{k} & -I_{k}\end{array}\right]\left(\begin{array}{l}u \\ v\end{array}\right)=\left(\begin{array}{r}H^{\mathrm{T}}(u-v) \\ -H^{\mathrm{T}}(u-v)\end{array}\right)$.

For $1 \leq i \leq k$ follows that

$z_{i}\left(M^{\mathrm{T} z}\right)_{i}=u_{i}\left(M^{\mathrm{T} z}\right)_{i}=u_{i}\left[H^{\mathrm{T}}(u-v)\right]_{i}$

and for $k+1 \leq i \leq 2 k$

$z_{i}\left(M^{\mathrm{T}} z\right)_{i}=v_{i-k}\left(M^{\mathrm{T}} z\right)_{i}=v_{i-k}\left[-H^{\mathrm{T}}(u-v)\right]_{i-k}$
And so

$u_{i}\left[H^{\mathrm{T}}(u-v)\right]_{i} \leq 0$ and $-v_{i}\left[-H^{\mathrm{T}}(u-v)\right]_{i} \leq 0$

for all $i=1,2, \ldots k$,

or

$(u-v)_{i}\left[H^{\mathrm{T}}(u-v)\right]_{i} \leq 0$ for all $i=1,2, \ldots, k$.

Because $H$ is a $\boldsymbol{P}$-matrix, it follows from Theorem 3.3 .4 of Cottle et al. (1992), that $u-v=0$. So

$M^{\mathrm{T}} z=\left(\begin{array}{r}H^{\mathrm{T}}(u-v) \\ -H^{\mathrm{T}}(u-v)\end{array}\right)=0 \Rightarrow z_{i}\left(M^{\mathrm{T}} z\right)_{i}=0$

for all $i=1,2, \ldots, 2 k$.

Lemma 10. Let $H$ and $M$ as in Lemma 9. The $\operatorname{LCP}(q, M)$ is solvable whenever it is feasible.

Proof. (In the proof of this Lemma, we use the terminology and notation from Cottle et al., 1992.) From Lemma 9 it follows that $M$ is a row sufficient matrix and hence, according to Corollary 3.5.5 in Cottle et al. (1992), a $\boldsymbol{Q}_{0}$-matrix. By the definition of $\boldsymbol{Q}_{0}$-matrices, this means that $\operatorname{LCP}(q, M)$ is solvable whenever it is feasible.

Lemma 11. If for some real $s>0, G(s)$ is a $\boldsymbol{P}$-matrix, then the $L C P(q(s), M(s))$, with $q(s)$ and $M(s)$ as defined in Eq. (20), is solvable.

Proof. Assume that for some real $s>0, G(s)$ is a $\boldsymbol{P}$-matrix. Then also $G^{-1}(s)$ is a $\boldsymbol{P}$-matrix (Lemma 7). According to Lemma 10, it is sufficient to show that a feasible vector pair $Y(s), U(s)$ exists for this $s$. Let $Y_{a}(s)=\left(T(s) x_{0}\right)^{+} \geq 0$ be the non-negative part of vector $T(s) x_{0}$ and let $Y_{b}(s)=\left(T(s) x_{0}\right)^{-} \geq 0$ be the non-positive part of this vector. Substituting this vector

$\left(\begin{array}{l}Y_{a}(s) \\ Y_{b}(s)\end{array}\right)=\left(\begin{array}{l}\left(T(s) x_{0}\right)^{+} \\ \left(T(s) x_{0}\right)^{-}\end{array}\right)$

in Eq. (18) we find

$$
\begin{aligned}
U_{a}(s)= & -G^{-1}(s) T(s) x_{0}+\frac{1}{s} d_{1}+G^{-1}(s)\left(T(s) x_{0}\right)^{+} \\
& -G^{-1}(s)\left(T(s) x_{0}\right)^{-} \\
= & -G^{-1}(s) T(s) x_{0}+\frac{1}{s} d_{1}+G^{-1}(s) T(s) x_{0} \\
= & \frac{1}{s} d_{1} \geq 0
\end{aligned}
$$

and

$$
\begin{aligned}
U_{b}(s)= & G^{-1}(s) T(s) x_{0}+\frac{1}{s} d_{2}-G^{-1}(s)\left(T(s) x_{0}\right)^{+} \\
& +G^{-1}(s)\left(T(s) x_{0}\right)^{-}
\end{aligned}
$$




$$
\begin{aligned}
& =G^{-1}(s) T(s) x_{0}+\frac{1}{s} d_{2}-G^{-1}(s) T(s) x_{0} \\
& =\frac{1}{s} d_{2} \geq 0 .
\end{aligned}
$$

So the $\operatorname{LCP}(q(s), M(s))$ is feasible.

After these preliminary lemmas, we now obtain our first main result.

Theorem 12. If for some real $s>0, G(s)$ is a P-matrix, then the $L C P(q(s), M(s))$ has a unique solution $U=\left(U_{a}^{\mathrm{T}}, U_{b}^{\mathrm{T}}\right)^{\mathrm{T}}, Y=\left(Y_{a}^{\mathrm{T}}, Y_{b}^{\mathrm{T}}\right)^{\mathrm{T}}$. This solution is such that $Y_{a}^{\mathrm{T}} Y_{b}=0$.

Proof. Assume that there exists an $s>0$ for which $G(s)$ is a $\boldsymbol{P}$-matrix, then we know from Lemma 11 that the $\operatorname{LCP}(q(s), M(s))$ has a solution. We will now prove that this solution is unique.

Assume that both

$$
\left(\begin{array}{l}
U_{a} \\
U_{b}
\end{array}\right),\left(\begin{array}{l}
Y_{a} \\
Y_{b}
\end{array}\right) \text { and }\left(\begin{array}{c}
\tilde{U}_{a} \\
\tilde{U}_{b}
\end{array}\right),\left(\begin{array}{c}
\tilde{Y}_{a} \\
\tilde{Y}_{b}
\end{array}\right)
$$

are solutions to the $\operatorname{LCP}(q(s), M(s))$

From Lemma 8 follows that $M(s)$ satisfies condition (c) of Theorem 3.4 .4 of Cottle et al. (1992). Therefore $\left(Y_{a}^{\mathrm{T}}, Y_{b}^{\mathrm{T}}\right)^{\mathrm{T}}$ and $\left(\tilde{Y}_{a}^{\mathrm{T}}, \tilde{Y}_{b}^{\mathrm{T}}\right)^{\mathrm{T}}$ satisfy

$M(s)\left[\begin{array}{l}Y_{a}-\tilde{Y}_{a} \\ Y_{b}-\tilde{Y}_{b}\end{array}\right]=\left[\begin{array}{rr}G^{-1}(s) & -G^{-1}(s) \\ -G^{-1}(s) & G^{-1}(s)\end{array}\right]\left[\begin{array}{l}Y_{a}-\tilde{Y}_{a} \\ Y_{b}-\tilde{Y}_{b}\end{array}\right]=0$

or

$G^{-1}(s)\left(\left(Y_{a}-\tilde{Y}_{a}\right)-\left(Y_{b}-\tilde{Y}_{b}\right)\right)=0$.

The matrix $G(s)$ was assumed to be a $\boldsymbol{P}$-matrix, and so we have

$Y_{a}-\tilde{Y}_{a}=Y_{b}-\tilde{Y}_{b}$

Now, define a vector $\Delta \in \mathbb{R}^{m}$ by

$\Delta_{i}=\left(Y_{a}\right)_{i}-\left(\tilde{Y}_{a}\right)_{i}=\left(Y_{b}\right)_{i}-\left(\tilde{Y}_{b}\right)_{i}, \quad i=1,2, \ldots, m$.

This results in

$$
\begin{gathered}
\left(Y_{a}\right)_{i}\left(Y_{b}\right)_{i}=\left(\tilde{Y}_{a}\right)_{i}\left(\tilde{Y}_{b}\right)_{i}+\left(\tilde{Y}_{a}+\tilde{Y}_{b}\right)_{i} \Delta_{i}+\Delta_{i}^{2} \\
i=1,2, \ldots, m .
\end{gathered}
$$

Because $Y_{a}=(\bar{Y})^{+}$, and $Y_{b}=(\bar{Y})^{-}$, we have $Y_{a}^{\mathrm{T}} Y_{b}=0$ and also $\tilde{Y}_{a}^{\mathrm{T}} \tilde{Y}_{h}=0$. Substituting this in Eq. (21), results in

$\left(\tilde{Y}_{a}+\tilde{Y}_{b}\right)_{i} \Delta_{i}+\Delta_{i}^{2}=0$

so

$\Delta_{i}=-\left(\tilde{Y}_{a}+\tilde{Y}_{b}\right)_{i}$ or $\Delta_{i}=0$.
Both possibilities result in $\Delta_{i}=0$ for $i=1,2, \ldots, m$, because in the first case we get

$\left(Y_{a}\right)_{i}=\left(\tilde{Y}_{a}\right)_{i}-\left(\tilde{Y}_{a}+\tilde{Y}_{b}\right)_{i}=-\left(\tilde{Y}_{b}\right)_{i}$,

$\left(Y_{b}\right)_{i}=\left(\tilde{Y}_{h}\right)_{i}-\left(\tilde{Y}_{a}+\tilde{Y}_{b}\right)_{i}=-\left(\tilde{Y}_{a}\right)_{i}$

and from the non-negativeness of $\left(Y_{a}\right)_{i},\left(Y_{b}\right)_{i},\left(\tilde{Y}_{a}\right)_{i}$ and $\left(\tilde{Y}_{b}\right)_{i}$ follows $\Delta_{i}=0$ for $i=1,2, \ldots, m$. We conclude that $Y_{a}=\tilde{Y}_{a}$ and $Y_{b}=\tilde{Y}_{b}$, and, consequently,

$$
\begin{aligned}
{\left[\begin{array}{l}
U_{a} \\
U_{b}
\end{array}\right] } & =q(s)+M(s)\left[\begin{array}{c}
Y_{a} \\
Y_{b}
\end{array}\right] \\
& =q(s)+M(s)\left[\begin{array}{c}
\tilde{Y}_{a} \\
\tilde{Y}_{b}
\end{array}\right]=\left[\begin{array}{c}
\tilde{U}_{a} \\
\tilde{U}_{b}
\end{array}\right] .
\end{aligned}
$$

After having provided in Theorem 12 sufficient conditions for unique solvability of the $\operatorname{LCP}(q(s), M(s))$ for sufficiently large $s \in \mathbb{R}$, we now turn to the existence and uniqueness of solutions to the original complementarity system (2)-(6). This is done via the Rational Complementarity Problem (RCP), as introduced initially by Van der Schaft and Schumacher (1998), and generalized in Heemels et al. (1997):

Rational Complementarity Problem $(\operatorname{RCP}(\tilde{q}(s), \tilde{M}(s))$. Let $\tilde{q}(s) \in \mathbb{R}^{k}(s)$ and $\tilde{M}(s) \in \mathbb{R}^{k \times k}(s)$ be given.

Find rational functions $Y(s)$ and $U(s)$ such that the equalities

$U(s)=\tilde{q}(s)+\tilde{M}(s) Y(s)$ and $U^{\mathrm{T}}(s) Y(s)=0$

hold for all $s$, and that there exists an $\tilde{s} \in \mathbb{R}_{+}$such that for all real $s \geq \tilde{s}$ we have

$U(s) \geq 0, \quad Y(s) \geq 0$.

For the $\operatorname{RCP}(q(s), M(s))$, with $q(s)$ and $M(s)$ as in (20), we can prove the following main result.

Theorem 13. If $G(s)$ is a $\boldsymbol{P}$-matrix for all real $s \geq s_{0}$ for some $s_{0} \in \mathbb{R}_{+}$, then the $\operatorname{RCP}(q(s), M(s))$ has a unique solution

$U(s)=\left(\begin{array}{c}U_{a}(s) \\ U_{b}(s)\end{array}\right), \quad Y(s)=\left(\begin{array}{c}Y_{a}(s) \\ Y_{b}(s)\end{array}\right)$

with $U(s), Y(s), \bar{U}(s)=\frac{1}{2}\left(U_{a}(s)+U_{b}(s)\right)-(1 / s)\left(d_{1}-d_{2}\right)$ and $Y(s)=Y_{a}(s)-Y_{b}(s)$ strictly proper rational functions.

Proof. The fact that the $\operatorname{RCP}(q(s), M(s))$ has a unique solution $U(s), Y(s)$ follows immediately from the rationality of $M(s)$ and $q(s)$ and Heemels et al. (1998), where it is shown that the $\operatorname{LCP}(q(s), M(s))$ for sufficiently large real $s$ has a unique solution if and only if $\operatorname{RCP}(q(s), M(s))$ has a unique solution. Note that $\bar{U}(s)$ and $\bar{Y}(s)$ are rational functions of $s$ because $U_{a}(s), U_{b}(s), Y_{a}(s)$ and $Y_{b}(s)$ are rational functions. From Eq. (15) and the conditions 
$U_{a}(s) \geq 0, U_{b}(s) \geq 0$ for sufficiently large $s$, we see that $-\frac{1}{s} d_{1} \leq \bar{U}(s) \leq \frac{1}{s} d_{2}$ for sufficiently large $s$.

From this we conclude that $\bar{U}(s)$ is a strictly proper rational function. Consequently, also $U_{a}(s)=(1 / s) d_{1}+\bar{U}(s)$ and $U_{b}(s)=(1 / s) d_{2}-\bar{U}(s)$ are strictly proper rational functions. Now, consider Eq. (14). The matrix $C\left(s I_{n}-A\right)^{-1} B+D$ is a proper matrix, $C\left(s I_{n}-A\right)^{-1}$ is a strictly proper matrix and $\bar{U}(s)$ is strictly proper. We immediately conclude that $\bar{Y}(s)$ is strictly proper.

Finally, $Y_{a}(s)$ and $Y_{b}(s)$ satisfy $\bar{Y}(s)=Y_{a}(s)-Y_{b}(s)$ and $Y_{a}^{\mathrm{T}}(s) Y_{b}(s)=0$. From the last equality follows that

$\left(Y_{a}(s)\right)_{i} \equiv 0$, or $\left(Y_{b}(s)\right)_{i} \equiv 0, \quad i=1,2, \ldots, m$

and so

$(\bar{Y}(s))_{i}=\left(Y_{a}(s)\right)_{i}$ or $(\bar{Y}(s))_{i}=-\left(Y_{b}(s)\right)_{i}, \quad i=1,2, \ldots, m$.

We conclude that $Y_{a}(s)$ and $Y_{b}(s)$ are strictly proper.

Finally, using the correspondence between strictly proper rational functions and real-analytic time functions as given in Eqs. (12) and (13), it follows (see also Heemels et al., 1998) that if the $\operatorname{RCP}(q(s), M(s))$ has a unique solution for all $q(s)$ as in Eq. (20) then the complementarity system (2)-(6) has for every fixed initial state $x_{0}$ a unique solution on some interval $[0, \varepsilon[, \varepsilon>0$, with $u(t), y(t), \bar{u}(t)$ and $\bar{y}(t)$ real-analytic functions of $t$. This implies that also the state $x(t)$ is real-analytic on $\left[0, \varepsilon\left[\right.\right.$. Now define $t_{1}$ as the maximal $\varepsilon$ such that $u(t), y(t)$ is a solution to Eqs. (2), (4)-(6). If $t_{1}=\infty$ then this means that there is a global solution from $x_{0}$ corresponding to one location (discrete state) of the relay system. If $t_{1}<\infty$, then this means that $t_{1}$ is a switching time, where we have to switch to another location (mode). Define

$x_{1} \stackrel{\text { Def }}{=} \lim _{t \uparrow t_{1}} x(t)$.

Then also from $x_{1}$ there exists a unique analytic solution of Eqs. (2), (4)-(6) on some interval $\left[t_{1}, t_{2}\right.$ [ of maximal length (with $t_{2} \leq \infty$ ). If $t_{2}<\infty$ then $t_{2}$ is the next switching time, and we define $x_{2}=\lim _{t \uparrow_{2}} x(t)$ as the initial condition for the next mode of operation of the relay system. Repeating this process we have obtained the following conclusion (see Fig. 5 for an illustration).

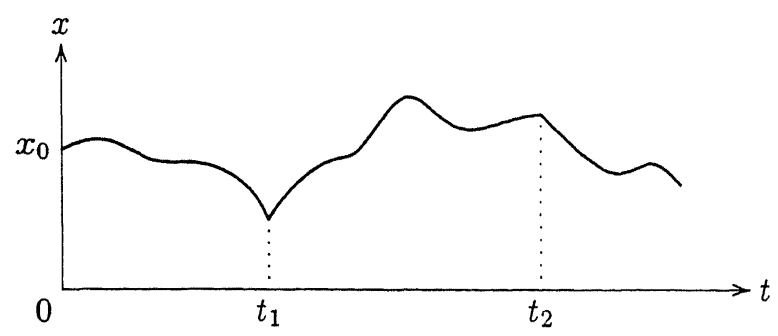

Fig. 5. The state $x(t)$.
Theorem 14. Consider the relay system given in Fig. 2. Assume that the transfer matrix $G(s)=C\left(I_{n} s-A\right)^{-1}$ $B+D$ is a P-matrix for $s \in \mathbb{R}$ sufficiently large. Then from every initial condition $x_{0}$ and initial time $t_{0}=0$ there exists a unique solution $\bar{u}(t), x(t), \bar{y}(t), t \geq 0$, such that $x(t)$ is a continuous function of $t$. Furthermore, this unique solution is piecewise real-analytic, in the sense that there exists a countable number of switching times $t_{i}$ such that $\bar{u}(t), x(t), \bar{y}(t)$ is real analytic on every time-interval $\left[t_{i}, t_{i+1}[\right.$.

Remark 15. Note that we have not excluded the possibility of existence of a finite accumulation point of the switching instants $t_{i}$. Nevertheless, "deadlock" is excluded also at such an accumulation point, since there exists a solution from the state reached at the accumulation point. An example of a finite accumulation point of switching instants is provided by the following relay system, which is derived by reversing time from an example of nonuniqueness of solutions in Filippov (1988):

$\dot{x}_{1}(t)=u_{1}(t)-2 u_{2}(t), \quad y_{1}(t)=x_{1}(t)$,

$\dot{x}_{2}(t)=2 u_{1}(t)+u_{2}(t), \quad y_{2}(t)=x_{2}(t)$

with,

$$
\begin{array}{ll}
u_{i}(t)=-1 & \text { if } y_{i}(t)>0 \\
u_{i}(t)=1 & \text { if } y_{i}(t)<0 \quad i=1,2 . \\
-1 \leq u_{i}(t) \leq 1 & \text { if } y_{i}(t)=0
\end{array}
$$

It can be verified that

$\frac{\mathrm{d}}{\mathrm{d} t}\left(\left|x_{1}(t)\right|+\left|x_{2}(t)\right|\right)=-2$,

which means that from every initial condition the system converges in finite time to the origin. Since solutions cannot arrive at the origin without going through an infinite number of mode switches, this means there is an accumulation of event times.

Remark 16. The LCP formulation of mechanical systems with Coulomb friction as employed in Lötstedt (1981) and Glocker and Pfeiffer (1993) is different from ours in at least two aspects. First, our formulation as a complementarity system as in Section 2 is different from Lötstedt (1981) and Glocker and Pfeiffer (1993). Secondly, in the present section we have transformed the complementarity conditions in the time-domain, via the RCP, to a simple $\mathrm{LCP}(q(s), M(s))$ for $s$ large enough. As a result the complexity of the LCP to be solved in our formulation is much less than in Lötstedt (1981) and Glocker and Pfeiffer (1993). Therefore, our LCP formulation does not seem to suffer from the drawbacks as mentioned in Cellier et al. (1993). 
Remark 17. Even if the solution is unique, we note, as in Van der Schaft and Schumacher $(1996,1998)$ that the corresponding mode need not be unique. (In these particular cases the input-output values $\left(\bar{u}_{i}, \bar{y}_{i}\right)$ remain at one of the two corners of the $i$-th relay characteristic for some $i$.)

Remark 18. It has been shown in this section that the condition of $G(s)$ being a $\boldsymbol{P}$-matrix for real $s$ large enough is sufficient for uniqueness of solutions for arbitrary initial conditions $x_{0}$. An interesting and important question is how close this condition is to being necessary as well.

It can be shown (starting from Theorem 3) that the $\operatorname{LCP}(q(s), M(s))$ for fixed real $s>0$ has a unique solution $U=\left(U_{a}^{\mathrm{T}}, U_{b}^{\mathrm{T}}\right)^{\mathrm{T}}, Y=\left(Y_{a}^{\mathrm{T}}, Y_{b}^{\mathrm{T}}\right)^{\mathrm{T}}$ for all $q \in \mathbb{R}^{n}$ if and only if $G(s)$ is a $\boldsymbol{P}$-matrix. However, in the $\operatorname{LCP}(q(s), M(s))$ the vector $q(s)$ has the specific structure (16), with $x_{0}$ being any initial condition. This implies that the rational vectors $q(s)$ in the $\operatorname{RCP}(q(s), M(s))$ obtained by letting $x_{0}$ range through $\mathbb{R}^{n}$ are not arbitrary. Thus the necessity of $G(s)$ being a $\boldsymbol{P}$-matrix for uniqueness of solutions for all $x_{0}$ is not guaranteed. Indeed, in the following example $G(s)$ is only a $\boldsymbol{P}_{0}$-matrix, while uniqueness of solutions for all $x_{0}$ does hold.

\section{Example 19.}

$\dot{x}_{1}(t)=-u_{2}(t) \quad y_{1}(t)=x_{1}(t)$,

$\dot{x}_{2}(t)=u_{1}(t), \quad y_{2}(t)=x_{2}(t)$

with,

$$
\begin{array}{ll}
u_{i}(t)=-1 & \text { if } y_{i}(t)>0 \\
u_{i}(t)=1 & \text { if } y_{i}(t)<0 \quad i=1,2 . \\
-1 \leq u_{i}(t) \leq 1 & \text { if } y_{i}(t)=0
\end{array}
$$

It can be easily seen that this system has a unique solution for every initial condition $x_{0}$, while

$$
G(s)=\left(\begin{array}{rr}
0 & -\frac{1}{s} \\
\frac{1}{s} & 0
\end{array}\right)
$$

is only a $\boldsymbol{P}_{0}$-matrix.

We conjecture that $G(s)$ being a $\boldsymbol{P}_{0}$-matrix is a necessary condition for uniqueness of solutions $U$ and $Y$ for all $x_{0}$.

Another problem concerning uniqueness of solutions is that even if solutions $U=\left(U_{a}^{\mathrm{T}}, U_{b}^{\mathrm{T}}\right)^{\mathrm{T}}, Y=\left(Y_{a}^{\mathrm{T}}, Y_{b}^{\mathrm{T}}\right)^{\mathrm{T}}$ and $\bar{U}, \bar{Y}$ may not be unique, the state-space solution $x$ may still be unique, as was kindly pointed out to us by an anonymous reviewer. A typical example is the case of two relays in parallel, where the total input may be unique, but not its distribution over the two relays. (In this case, the $B$ and the $C$ matrix in (2) are not injective, respectively, surjective.)
It is of interest to find necessary and sufficient conditions for uniqueness of the solution $x(t)$. Again, we conjecture that $G(s)$ being a $\boldsymbol{P}_{0}$-matrix is a necessary condition.

Remark 20. In switching control schemes such as (see Eq. (1)) $u=1$ for $y<0$ and $u=-1$ for $y>0$, the dynamics for $y=0$ is usually deliberately left open. Indeed, the dynamics for $y=0$ will be seen as the limit of a chattering behavior around the level set $y=0$ in the state space (rapid switchings between $u=1$ and $u=-1$ ). In this context Filippov's equivalent control or convex definition (equivalent for systems linear in $u$ ) is employed. Note however (see e.g. Filippov, 1988) that this assumes that the velocity vector $\dot{x}$ for $u=1$ points for $y$ close to 0 into the direction of the subset of the state space defined by $y>0$, and the velocity vector $\dot{x}$ for $u=-1$ points for $y$ close to 0 into the direction of the subset of the state space defined by $y<0$. (Otherwise we do not obtain chattering.)

On the other hand, if $G(s)$ is a $P$-matrix then based upon Theorem 14, we may look at the situation in the following manner. Consider for simplicity the single-relay case with $D=0$. Let $x_{0}$ be an initial condition with $y=0$. By Theorem 14 there exists a unique solution from $x_{0}$ corresponding either to $u=-1, u=1$ or $y=0$ (in the equivalent control sense). If the unique solution corresponds to $u=-1$ or $u=1$ then obviously we are done. Now consider the case that the unique solution only corresponds to the mode $y=0$. Then by the very fact that there is no solution corresponding to $u=1$ or $u=-1$ it follows that the velocity vector $\dot{x}$ for $u=1$ points for $y=0$ into the direction of the set $y>0$, and for $u=-1$ into the direction of the set $y<0$. Thus Filippov's equivalent control definition does make sense. This discussion can be extended to the general case of $G(s)$ being a $\boldsymbol{P}$-matrix.

Our theoretical results suggest the following approach to simulation of relay systems (see Mattsson, 1996; Cellier et al., 1993) for a clear discussion of the basic issues in simulation of such systems, and Heemels et al., 1997 for a similar approach to simulation of complementarity systems arising from ideal diode characteristics).

Let $x_{0}$ be the initial condition and $t_{0}$ the initial time. Consider $M(s), q(s)$ as determined in Eq. (20), and solve the $\operatorname{LCP}(q(s), M(s))$ for fixed real $s$ large enough. This yields a unique solution $\bar{U}(s), \bar{Y}(s)$, with index sets $I_{1}, I_{2} \subset\{1,2, \ldots, m\}$ with $I_{1} \cap I_{2}=\emptyset$ (not necessarily uniquely determined), such that

$$
\begin{array}{ll}
\bar{Y}_{i}(s)>0, & i \in I_{1}, \\
\bar{Y}_{i}(s)<0, & i \in I_{2}, \\
\bar{Y}_{i}(s)=0, & i \notin I_{1} \cup I_{2} .
\end{array}
$$


Based on these index sets we consider the system of differential-algebraic equations (DAEs)

$\dot{x}=A x+B \bar{u}, \quad x\left(t_{0}\right)=x_{0}$,

$0=(C x+D \bar{u})_{i}, \quad i \notin I_{1} \cup I_{2}$,

$\bar{u}_{i}=-\left(d_{1}\right)_{i}, \quad i \in I_{1}$,

$\bar{u}_{i}=\left(d_{2}\right)_{i}, \quad i \in I_{2}$.

If we have chosen $s$ large enough then it follows from Theorems 13 and 14 that the unique solution of Eq. (23) satisfies

$\bar{y}_{i}(t) \geq 0, \quad i \in I_{1}$,

$\bar{y}_{i}(t) \leq 0, \quad i \in I_{2}$,

$\bar{u}_{i}(t) \in\left[-\left(d_{1}\right)_{i},\left(d_{2}\right)_{i}\right], \quad i \notin I_{1} \cup I_{2}$,

for $t \in\left[t_{0}, t_{0}+\varepsilon[, \varepsilon>0\right.$. Hence we may numerically simulate the set of DAEs (23), while monitoring inequalities (24). (Obviously, the simulation of the DAEs (23) may not be an easy task, and, for example, we may wish to convert Eq. (23) into a set of explicit differential equations first.) If for some time $t_{1}>t_{0}$ inequalities (24) are going to be violated (event detection), then for this switching time $t_{1}$ we again consider the $\operatorname{LCP}(q(s), M(s))$, for fixed real $s$ large enough, with now $q(s)$ determined as in Eq. (18) by

$x\left(t_{1}\right)=\lim _{t \uparrow t_{1}} x(t)$.

This will yield again a unique solution $\bar{u}^{\prime}(s), \bar{y}^{\prime}(s)$, with index sets $I_{1}^{\prime}, I_{2}^{\prime}$ defining as in Eq. (23) a set of DAEs, which then can be simulated, etc.

Note that the $\operatorname{LCP}(q(s), M(s))$, even for large $m$, admits efficient solution routines (see e.g. Cottle et al., 1992), and so the above strategy seems to offer a convenient way to handle simulation of relay systems.

Obviously, the weak point in the suggested strategy, is that we do not know beforehand "how large" $s$ has to be chosen for the $\operatorname{LCP}(q(s), M(s))$ at every switching time. If we take $s$ too small, then we will select different index sets $I_{1}, I_{2}$, and the solution of the DAE's (23) will not satisfy Eq. (24)

On the other hand, after solving the $\operatorname{LCP}(q(s), M(s))$ for some $s \in \mathbb{R}$, and thus obtaining index sets $I_{1}$ and $I_{2}$, it is possible to check algebraically whether these index sets $I_{1}, I_{2}$ provide a solution for the $\operatorname{RCP}(q(s)$, $M(s)$ ), or equivalently (using the aforementioned correspondence between strictly proper rational func- tions in $s$ and real-analytic time-functions, a solution for Eq. (24).

\section{Examples}

Example 21 (System (1)). Consider again the system (1) described in Section 1. For this system $G(s)$ is given by

$G(s)=\left[\begin{array}{ll}0 & 1\end{array}\right]\left[\begin{array}{rr}s & -1 \\ 1 & s\end{array}\right]^{-1}\left[\begin{array}{r}0 \\ -1\end{array}\right]=-\frac{s}{s^{2}+1}$.

Obviously $G(s)$ does not satisfy the conditions of Theorem 13, and so, uniqueness of solutions is not guaranteed, in accordance with the further treatment of this system in Section 1. If the sign in front of $u$ in the first equation of Eq. (1) is reversed, then $G(s)=s /\left(s^{2}+1\right)$ does satisfy the conditions of Theorem 13 , and so there is only one solution from every initial state.

Example 22 (Coulomb friction). Consider the system with multiple Coulomb friction as given in Fig. 6 (see also Glocker and Pfeiffer, 1993). In this figure we see two rigid blocks that are connected to a fixed wall by springs. The block on the bottom has mass $m_{1}>0$, the block on top has mass $m_{2}>0$. The blocks make contact at the points 1 and 2. In these points Coulomb friction forces $F_{c 1}$ and $F_{c 2}$ act. By definition $F_{c 2}$ is the Coulomb friction as it is acting on block 2 . There is of course also an equal, but opposite friction-force acting on block 1 . Let $x_{1}$ represent the deviation of the bottom block from its equilibrium position (no elongations of the spring $k_{1}$ ). Let $x_{2}$ represent the position of the top block, relative to the bottom block. This coordinate is chosen in such a manner that the system is in equilibrium for $x_{1}=x_{2}=0$. Let $x_{3}$ and $x_{4}$ denote the corresponding velocities. The Coulomb friction characteristic of $F_{c 1}$ is as in Fig. 1 with $e=F_{c 1}$ and $f$ the velocity $\dot{x}_{1}$ (while $c_{1}=c_{2}=1$ ). Similarly for the Coulomb friction $F_{c 2}$ with regard to the velocity $\dot{x}_{2}$.

The system can be described by the equations of a relay system as in Section 3. The plant dynamics described

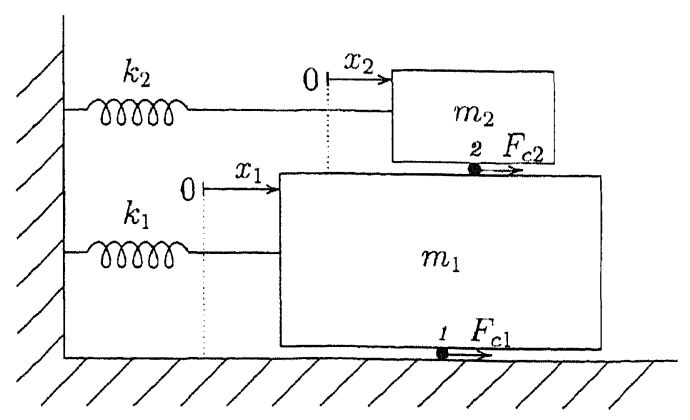

Fig. 6. Two blocks with Coulomb friction 
by Eq. (2) are for this system given by

$$
\dot{x}(t)=\left[\begin{array}{cccc}
0 & 0 & 1 & 0 \\
0 & 0 & 0 & 1 \\
-\frac{k_{1}}{m_{1}} & 0 & 0 & 0 \\
\frac{k_{1}}{m_{1}}-\frac{k_{2}}{m_{2}} & -\frac{k_{2}}{m_{2}} & 0 & 0
\end{array}\right] x(t)
$$$$
+\left[\begin{array}{cc}
0 & 0 \\
0 & 0 \\
\frac{1}{m_{1}} & -\frac{1}{m_{1}} \\
-\frac{1}{m_{1}} & \frac{1}{m_{1}}+\frac{1}{m_{2}}
\end{array}\right]\left[\begin{array}{l}
\bar{u}_{1}(t) \\
\bar{u}_{2}(t)
\end{array}\right]
$$

$$
\left(\bar{u}_{i}=-F_{c i}, i=1,2\right) \text {, }
$$

$\bar{y}(t)=\left[\begin{array}{llll}0 & 0 & 1 & 0 \\ 0 & 0 & 0 & 1\end{array}\right] x(t)$

with

$$
\begin{array}{ll}
\bar{u}_{i}(t)=-1 & \text { if } \bar{y}_{i}(t)>0 \\
\bar{u}_{i}(t)=1 & \text { if } \bar{y}_{i}(t)<0, \\
-1 \leq \bar{u}_{i}(t) \leq 1 & \text { if } \bar{y}_{i}(t)=0 .
\end{array}
$$

For this relay system the transfer matrix $G(s)$ is given by

$G(s)=\left[\begin{array}{cc}\frac{s}{m_{1} s^{2}+k_{1}} & -\frac{s}{m_{1} s^{2}+k_{1}} \\ -\frac{s}{m_{1} s^{2}+k_{1}} & \frac{s\left(\left(m_{1}+m_{2}\right) s^{2}+k_{1}+k_{2}\right)}{\left(m_{1} s^{2}+k_{1}\right)\left(m_{2} s^{2}+k_{2}\right)}\end{array}\right]$.

This matrix is a $\boldsymbol{P}$-matrix if and only if the principal minors of this matrix are positive:

$\frac{s}{m_{1} s^{2}+k_{1}}>0$

and

$\frac{s\left(\left(m_{1}+m_{2}\right) s^{2}+k_{1}+k_{2}\right)}{\left(m_{1} s^{2}+k_{1}\right)\left(m_{2} s^{2}+k_{2}\right)}>0$

and

$\frac{s^{2}}{\left(m_{1} s^{2}+k_{1}\right)\left(m_{2} s^{2}+k_{2}\right)}>0$

(in fact, because the matrix $G(s)$ is symmetric, we may as well restrict (see Cottle et al., 1992) to the leading principal minors (25) and (27)). We immediately see that this is true for real $s$ large enough if $m_{1}>0$ and $m_{2}>0$. Thus (as is physically evident), the system has unique solutions if both $m_{1}$ and $m_{2}$ are positive.

\section{Conclusions}

Relay systems form a particular type of hybrid dynamical systems, where the discrete transition rules (from one mode to another) are not a priori given. For linear time-invariant relay systems we have shown, based on the formulation of a relay system as a complementarity system, that if the transfer matrix is a $P$-matrix (for real $s$ large enough) then the relay system has a unique solution that is continuous in the state; thereby specifying the discrete transition rules of the system. We have argued that our results suggest a promising approach to the simulation of relay systems, by solving at every switching time an LCP and simulating during the subsequent timeinterval a set of DAEs specified by the solution of this LCP. Further topics for research concern the generalization of the obtained results to linear dynamical systems containing arbitrary piecewise-linear characteristics, and the extension to nonlinear dynamics.

\section{Acknowledgements}

We thank the NWO (Dutch organization for scientific research) for making the participation of Y.J. Lootsma possible. We thank The Scientific and Technical Research Council of Turkey (TÜBİTAK) for awarding a NATO Science Fellowship to M. Kanat Çamlıbel which made his participation in this study possible. We thank the anonymous reviewers for insightful and constructive remarks on the first version of this paper. Also, the second author likes to thank Hans Schumacher for the collaboration which started the research presented in this paper.

\section{References}

Barton, P. I., \& Pantelides, C. C. (1994). Modeling of combined discrete/continuous processes, A.I.Ch.E. J., 41),966-979.

Brogliato, B. (1996). Nonsmooth impact mechanics - models, dynamics and control. Lecture Notes in Control and Information Sciences. Vol. 220. Berlin: Springer.

Cottle, R. W., Pang, J.-S., \& Stone, R. E. (1992). The lin'ar complementarity problem. Academic Press, Computer Sciences and Scientific Computing. New York: Academic Press.

Filippov, A. F. (1988). Differential equations with discontinuous righthand sides, Dordrecht: Kluwer Academic Press.

Glocker, Ch., \& Pfeiffer, F. (1993). Complementarity problems in multibody systems with planar friction, Arch. Appl. Mech., 63, 452-463.

Cellier, F. E., Elmquist, H., \& Otter, M. (1993). Object-oriented modelling of hybrid systems. Proc. European Simulation Symp., ESS'93 (pp. 31-41).

Heemels, W. P. M. H., Schumacher, J. M., \& Weiland, S. (1997). Linear complementarity systems. Report 97 1/01, Eindhoven University of Technology, Dept. of Electrical Engineering. Measurement and Control Systems, Eindhoven, The Netherlands.

Heemels, W. P. M. H., Schumacher, J. M., \& Weiland, S. (1998). The rational complementarity problem. Technical Report 98 1/02, 
Eindhoven University of Technology, Dept. of Electrical Engineering. Measurement and Control Systems, Eindhoven, The Netherlands.

Lötstedt, P. (1981). Coulomb friction in two-dimensional rigid body systems. ZAMM, 61, 605-615.

Mattsson, S. E. (1996). On object-oriented modelling of relays and sliding mode behaviour. Preprints IFAC 13th World Cong., F, 259-264.

Van der Schaft, A. J., \& Schumacher, J. M. (1996). The complementary slackness class of hybrid systems. Math. Control Signals Systems, 9 , 266-301.

Van der Schaft, A. J., \& Schumacher, J. M. (1998). Complementarity modeling of hybrid systems. IEEE Trans. Automat. Control, Special Issue on Hybrid Control Systems, 43, 483-490.

Seidman, T. I. (1995). Some limit problems for relays. Proc. 1st Congress of Nonlinear Analysts (Tampa, 1992) (pp. 787-796). Berlin: Springer.

Tsypkin, Ya. Z. (1984). Relay control systems. New York: Cambridge University Press.

Utkin, V. I. (1992). Sliding modes in control and optimization. Communications and Control Engineering Series, Berlin: Springer.

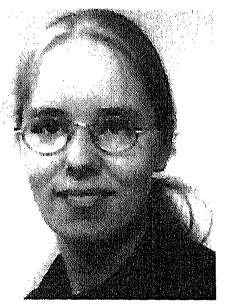

Yvonne J. Lootsma was born in Haskerland, The Netherlands, in 1974. She received the undergraduate degree in Applied Mathematics from the University of Twente, The Netherlands, in 1997, where she is currently a $\mathrm{PhD}$ candidate, sponsored by the NWO (Dutch organization for scientific research). Her research interests include nonlinear control systems and hybrid systems.

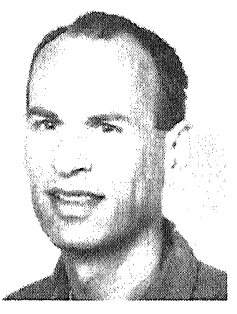

A.J. (Arjan) van der Schaft was born in Vlaardingen, The Netherlands, in 1955 . He received the undergraduate and $\mathrm{PhD}$ degrees in Mathematics from the University of Groningen, The Netherlands, in 1979 and 1983 , respectively.

In 1982 he joined the Faculty of Math. ematical Sciences, University of Twente Enschede, The Netherlands, where he is presently an Associate Professor. His research interests include the mathematical modelling of physical and engineering systems and the control of nonlinear and hybrid systems. He has served as an Associate Editor for Systems \& Control Letters, Journal of Nonlinear Science, and the IEEE Transactions on Automatic Control. He is the author of Systems Theoretic Descriptions of Physical Systems (Amsterdam, The Netherlands: CWI, 1984) and coauthor of Variational and Hamiltonian Control Systems (Berlin, Germany: Springer-Verlag, 1987) and Nonlinear Dynamical Control Systems (Berlin, Germany: SpringerVerlag, 1990), as well as the author of $L_{2}$-Gain and Passivity Techniques in Nonlinear Control (London, UK: Springer-Verlag, 1996) and the coauthor (with J.M. Schumacher) of An Introduction to Hybrid Systems (London, UK: Springer-Verlag, LNCIS, 1999, to appear).

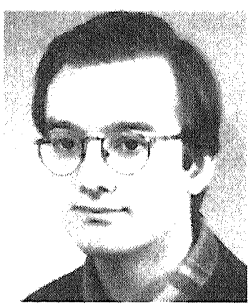

M. Kanat Çamlıbel received his B.Sc. and M.Sc. degrees in control and computer engineering from Istanbul Technical University, Turkey in 1991 and 1994, respectively. From 1991 to 1997, he worked as a teaching assistant at the same university. After being awarded a grant by The Scientific and Technical Research Council of Turkey (TÜBITAK), he moved to The Netherlands for $\mathrm{PhD}$ studies in 1997. His research interests include discontinuous dynamics and piecewise linear systems. 